\title{
Gradhiva
}

\section{Sandra Bornand et Cécile Leguy, Anthropologie des pratiques langagières}

Paris, Armand Colin, coll. « Sciences humaines et sociales », 2013

\section{Julien Bonhomme}

\section{OpenEdition}

\section{Journals}

Édition électronique

URL : http://journals.openedition.org/gradhiva/2999

DOI : 10.4000/gradhiva.2999

ISSN : 1760-849X

\section{Éditeur}

Musée du quai Branly Jacques Chirac

Édition imprimée

Date de publication : 1 février 2015

Pagination : 248-249

ISBN : 978-2-35744-075-3

ISSN : 0764-8928

\section{Référence électronique}

Julien Bonhomme, "Sandra Bornand et Cécile Leguy, Anthropologie des pratiques langagières »,

Gradhiva [En ligne], 21 | 2015, mis en ligne le 01 février 2015, consulté le 22 septembre 2020. URL: http://journals.openedition.org/gradhiva/2999; DOI : https://doi.org/10.4000/gradhiva.2999

Ce document a été généré automatiquement le 22 septembre 2020.

(c) musée du quai Branly 


\section{Sandra Bornand et Cécile Leguy, Anthropologie des pratiques langagières}

Paris, Armand Colin, coll. « Sciences humaines et sociales », 2013

Julien Bonhomme

\section{RÉFÉRENCE}

Sandra Bornand et Cécile Leguy, Anthropologie des pratiques langagières. Paris, Armand Colin, coll. « Sciences humaines et sociales », 2013.

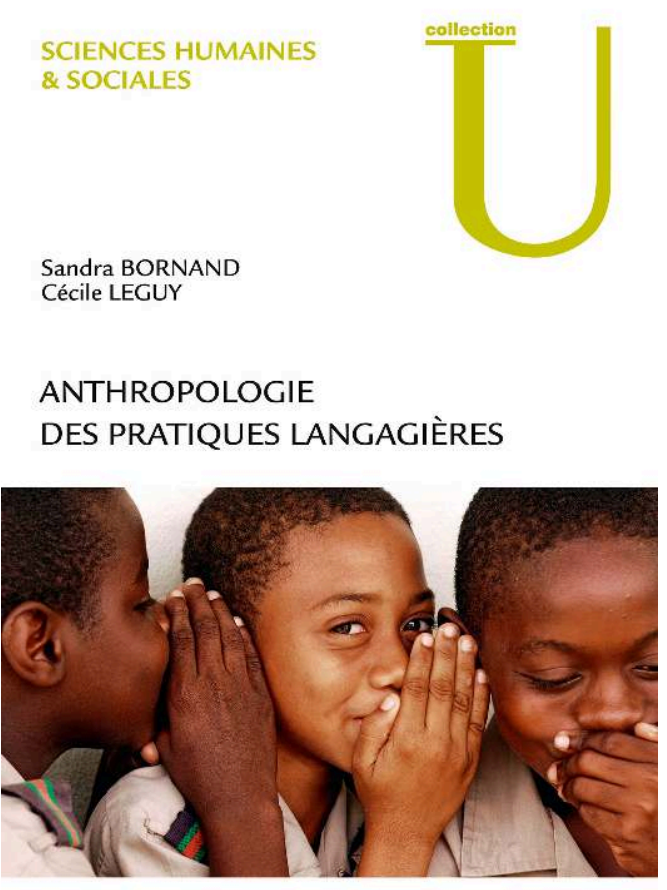

ARMAND COLIN 
1 En 1962, Dell Hymes, anthropologue et linguiste américain spécialiste des Indiens de la côte Nord-Ouest, publie un manifeste pour une " ethnographie de la parole » : il s'agit d'étudier l'articulation entre langue, culture et société dans la continuité des travaux de Franz Boas, David Sapir et Benjamin Lee Whorf, mais en mettant plus résolument l'accent sur l'analyse de pratiques langagières en contexte ${ }^{1}$. Deux ans plus tard, Hymes dirige avec le sociolinguiste John Gumperz un numéro spécial de la revue American Anthropologist consacré à l'« ethnographie de la communication ", nouveau nom de baptême qui met en avant le fait que la communication humaine ne se réduit pas à ses éléments verbaux ${ }^{2}$. Ce numéro, auquel contribuent des auteurs comme Erving Goffman et William Labov, joue un rôle fondateur dans la constitution d'un nouveau champ de recherches au carrefour des sciences sociales et des sciences du langage. Dix ans plus tard, deux étudiants de Hymes à l'université de Pennsylvanie, Richard Bauman et Joel Sherzer, font paraître un ouvrage collectif intitulé Explorations in the Ethnography of Speaking ${ }^{3}$, qui représente un premier aboutissement du programme lancé par leur maître.

2 Depuis lors, l'ethnographie de la communication s'est imposée comme le paradigme dominant en anthropologie linguistique et a suscité une tradition de recherche extrêmement féconde, en particulier aux États-Unis où elle est communément enseignée dans les départements d'anthropologie. Il existe en langue anglaise de nombreux ouvrages d'introduction à ce courant, parmi lesquels la remarquable trilogie d'Alessandro Duranti, professeur à UCLA et auteur d'un manuel, d'une anthologie et d'un companion consacrés à l'anthropologie linguistique ${ }^{4}$. En France, l'ethnographie de la communication n'a en revanche jamais bénéficié d'une telle reconnaissance académique, en dépit des travaux pionniers de Geneviève Calame-Griaule qui inaugure l'ethnolinguistique à la française dans les années $1960^{5}$. Certes, nombre d'anthropologues hexagonaux ont apporté des contributions originales à ce courant de recherche ${ }^{6}$. Mais il manquait encore cruellement un bon ouvrage d'introduction en français : le manuel de Duranti, qui existe pourtant en version italienne et espagnole, n'a jamais été traduit en France et les lecteurs francophones ne disposaient guère que des ouvrages d'Yves Winkin, au demeurant fort intéressants quoique un peu trop étroitement centrés sur l'école de Palo Alto7

3 Le livre de Sandra Bornand et Cécile Leguy consacré à l'anthropologie des pratiques langagières vient très opportunément combler ce quasi-vide. Ses auteurs sont toutes les deux ethnolinguistes et spécialistes de l'Afrique de l'Ouest ${ }^{8}$. Leur ouvrage se présente comme un manuel destiné aux étudiants de troisième cycle universitaire. Très pédagogique, il expose avec clarté et précision l'histoire, les théories, les concepts et les méthodes de l'ethnographie de la communication, et les illustre de nombreuses études de cas signalées par des encadrés. La première partie passe en revue deux des concepts centraux de ce courant de recherche : les notions de contexte et de communication. Le premier chapitre souligne le rôle précurseur de Bronislaw Malinowski qui, dans les annexes des Jardins de corail, proposait déjà une approche pragmatique du langage en pensant la signification comme l'effet produit par l'énoncé au sein de la situation d'énonciation'. Il aborde en outre la question épineuse de la délimitation du contexte et de l'échelle d'analyse (qui se pose en réalité à toutes les sciences sociales et pas seulement à l'anthropologie linguistique) : comment articuler contexte linguistique, situation d'énonciation et contexte socioculturel ou historique ? Le deuxième chapitre part de la critique du modèle télégraphique de la communication, trop mécaniste et 
linéaire, et insiste sur la nécessité d'élargir le concept de communication pour prendre en compte ses aspects para- et extralinguistiques.

4 La seconde partie de l'ouvrage s'intéresse aux pratiques langagières elles-mêmes. Le troisième chapitre porte sur la notion de parole: il présente le modèle élaboré par Hymes pour étudier les «événements de parole » (speech events), mais aborde aussi la question de l'oralité et de l'écriture. Le chapitre suivant est consacré aux arts de la parole et traite de la performance, de la composition, de la mémorisation et de la transmission des traditions orales. L'avant-dernier chapitre porte sur les situations d'interlocution en s'intéressant par exemple aux proverbes, aux joutes verbales et aux insultes rituelles. Le dernier chapitre prend parti «pour une anthropologie pragmatique et énonciative" (au prix cependant de quelques redondances avec le précédent chapitre, sur les insultes notamment). Il insiste sur l'importance des concepts de performativité et d'agentivité et souligne, à la suite de Malinowski, John Austin et Ruth Finnegan, que le langage ne fait pas uniquement l'objet d'un usage référentiel, mais constitue aussi une forme d'action sociale en soi ${ }^{10}$.

Pour juger d'un ouvrage d'introduction à l'anthropologie linguistique, le manuel de Duranti représente un point de comparaison incontournable. À cette aune, il faut bien reconnaître que le livre de Bornand et Leguy, deux fois moins épais que le "Duranti ", se révèle moins complet, quoique plus facile d'accès. Il se situe en deçà sur les aspects plus techniquement linguistiques (il n'y a par exemple aucune analyse grammaticale d'énoncés en langue vernaculaire), mais aussi sur la méthodologie : la question des choix de transcription est presque complètement passée sous silence, tandis que Duranti y consacre un chapitre entier. En revanche, le livre de Bornand et Leguy a ceci d'original qu'il présente sur un même pied l'anthropologie linguistique à l'américaine et l'ethnolinguistique à la française, alors que cette dernière est souvent minorée dans les manuels anglophones, quand elle n'est pas tout simplement ignorée : Duranti ne fait par exemple qu'une brève allusion à Calame-Griaule, dont l'ouvrage sur la parole chez les Dogon a pourtant été traduit en anglais en 1986. Bornand et Leguy connaissent quant à elles fort bien l'anthropologie linguistique américaine, mais elles mettent également en avant tout un ensemble de travaux d'auteurs de langue française. En définitive, ce livre possède sans aucun doute les qualités nécessaires pour devenir un manuel de référence et introduire à l'anthropologie linguistique et l'ethnographie de la communication un lectorat francophone encore trop peu au fait de ce courant de recherche.

\section{NOTES}

1. Dell Hymes, "The ethnography of speaking ", in Thomas Gladwin et William C. Sturtevant (éd.), Anthropology and Human Behavior. Washington, Anthropological Society of Washington, 1962 : 13-53.

2. John Gumperz et Dell Hymes (dir.), "The ethnography of communication ", American Anthropologist LXVI (6) 1964. Le numéro, augmenté de plusieurs articles, sera republié huit ans 
plus tard sous forme d'ouvrage : Directions in Sociolinguistics: The Ethnography of Communication. New York, Holt, Rinehart \& Winston, 1972.

3. Richard Bauman et Joel Sherzer (éd.), Explorations in the Ethnography of Speaking. Cambridge, Cambridge University Press, 1974.

4. Alessandro Duranti, Linguistic Anthropology. Cambridge, Cambridge University Press, 1997 ; id. (éd.), Linguistic anthropology: A Reader. Oxford, Blackwell, 2001 ; id. (éd.), A Companion to Linguistic Anthropology. Malden, Blackwell, 2004. Voir aussi Muriel Saville-Troike, The Ethnography of Communication. Baltimore, University Park Press, 1982; William Hanks, Language and Communicative Practices. Boulder, Westview Press, 1996; Christine Jourdan et Kevin Tuite, Language, Culture, and Society. Cambridge, Cambridge University Press, 2006.

5. Geneviève Calame-Griaule, Ethnologie et langage. La parole chez les Dogon. Paris, Gallimard, 1965 ; id. (éd.), Langage et cultures africaines. Essais d'ethnolinguistique. Paris, Maspero, 1977.

6. Voir par exemple Bertrand Masquelier et Jean-Louis Siran (éd.), Pour une anthropologie de l'interlocution: rhétoriques du quotidien. Paris, L'Harmattan, 2000 ; Aurore Monod-Becquelin et Philippe Erikson (éd.), Les Rituels du dialogue : promenades ethnolinguistiques en terres amérindiennes. Nanterre, Société d'ethnologie, 2000.

7. Yves Winkin, Anthropologie de la communication. De la théorie au terrain. Paris, Seuil, 2001 (1 ${ }^{\text {re }}$ édition 1996) ; id. (dir.), La Nouvelle Communication. Paris, Seuil, 1981.

8. Voir Cécile Leguy, Le Proverbe chez les Bwa du Mali. Parole africaine en situation d'énonciation. Paris, Karthala, 2001 ; Sandra Bornand, Le Discours du griot généalogiste chez les Zarma du Niger. Paris, Karthala, 2005.

9. Bronislaw Malinowski, Jardins de Corail. Paris, Maspero, 1974 ( $1^{\text {re }}$ éd. anglaise 1935).

10. Sur ce thème, voir aussi Carlo Severi et Julien Bonhomme (dir.), Paroles en actes. Paris, L'Herne, 2009.

\section{AUTEURS}

\section{JULIEN BONHOMME}

julienbonhomme@yahoo.fr 